\title{
Mineração
}

\section{Caracterização tecnológica de resíduos de pedreiras de quartzito da região de Ouro Preto/MG}

\author{
(Technological characterization of the residuals from a \\ quartzite quarry of Ouro Preto/MG)
}

\author{
Rosa Malena Fernandes Lima \\ Professora do PPGEM/DEMIN/UFOP.E-mail: rosa@demin.ufop.br \\ Alexandro Fortes Simões da Silva \\ Engenheiro de Minas. E-mail: alexandrofortes1@yahoo.com.br \\ Rubhia Marianna Maciel de Morais \\ Mestranda do PPGEM/UFOP.E-mail: rubiamorais@pop.com.br \\ José Aurélio Medeiros da Luz \\ Professor do PPGEM/DEMIN/UFOP.E-mail: jaurelio@demin.ufop.br
}

\section{Resumo}

Os quartzitos da serra do Taquaral em Ouro PretoMG são extraídos para serem usados como placa de pisos e revestimentos. Pelo fato $\mathrm{dE}$ a rocha ser bastante intemperizada e, por essa razão, ser friável, são geradas, nas frentes de lavra e no beneficiamento, um grande volume de resíduos de ampla distribuição granulométrica. Por essa razão, esse trabalho teve, por objetivo, caracterizar as areias provenientes das frentes de lavra dessas pedreiras, que são, normalmente, depositadas nas encostas dos morros. Através dos estudos realizados com três amostras, verificou-se a viabilidade técnica de utilização das mesmas na construção civil, na fundição e, após purificação por separação magnética de alta intensidade e flotação dos minerais portadores dos elementos químicos deletérios $\left(\mathrm{F}_{2} \mathrm{O}_{3}, \mathrm{Al}_{2} \mathrm{O}_{3}\right.$ e $\left.\mathrm{TiO}_{2}\right)$, vislumbra-se a possibilidade do uso dessas areias na indústria de vidro, o que é extremamente importante do ponto de vista social, econômico e ambiental.

Palavras-chave: Caracterização tecnológica de resíduos, areias de quartzito.

\begin{abstract}
Quartzites from Taquaral Hill at Ouro Preto - in the State of Minas Gerais - have been exploited for construction and ornamental purposes for decades. Until now, the rudimentary way to exploit and refine these weatherized rocks has produced, a large amount of residuals distributed in a broad size distribution range. This work aimed to characterizes the sand and debris from the mining operation, which are deposited downhill. Three samples were studied and it became evident that this debris can be used as sand in the civil construction and foundry industries. Also, after removing the impurities $\left(\mathrm{F}_{2} \mathrm{O}_{3}, \mathrm{Al}_{2} \mathrm{O}_{3}\right.$ e $\left.\mathrm{TiO}_{2}\right)$ by high intensity magnetic separation and froth flotation, it can be used in the glass industry, which is of social, economical and environmental interest.
\end{abstract}

Keywords: Waste technological characterization, quartzite sands. 


\section{Introdução}

O aproveitamento dos quartzitos da região de Ouro Preto como rocha ornamental é uma atividade econômica que vem sendo empreendida há décadas. A lavra é efetuada de modo muito rudimentar e em condições precárias. Normalmente, são produzidas placas de piso e revestimento. As placas destacadas e aproveitáveis são empilhadas para o transporte (o qual é parcialmente feito com muares). O baixo emprego de técnicas racionais modernas, somado ao fato de que somente uma parcela dos corpos quartzíticos ser utilizável para a confecção de placas para pisos e revestimentos, gera uma grande quantidade de resíduos, que são deixados nas encostas dos morros. Segundo as empresas produtoras, para a produção mensal atual de $1.500 \mathrm{~m}^{3}$, o volume total de rejeito solto é de aproximadamente $300.000 \mathrm{~m}^{3}$. No entanto, a atividade emprega diretamente 350 pessoas. A Figura 1 evidencia o enorme impacto ambiental causado pela atividade na região do morro do Taquaral (cerca de $3 \mathrm{~km}$ do centro de Ouro Preto, MG), onde se concentram as pedreiras de quartzito.

Lima e colaboradores (2005), através de microscopia ótica e microscopia eletrônica de varredura, determinaram a composição mineralógica dos fragmentos decimétricos de quartzito, depositados nas encostas dos morros nas proximidades das pedreiras. Os minerais identificados foram o quartzo (85 \%), micas (moscovita e cianita - 10\%), sericita (5\%). Os minerais opacos rutilo, zircão, hematita, goethita, calcopirita e prata constituem cerca de 2 \% da composição mineralógica desses quartzitos.

As areias de quartzo têm ampla aplicação industrial (na construção civil, fundição, indústria vidreira, etc.). As especificações de areias para a construção civil e para fundição baseiam-se, essencialmente, na granulometria, com especificações químicas menos rígidas. Para a indústria de vidro, as exigências, em termos de teores de sílica e de impurezas, são mais rigorosas, conforme mostrado na Tabela 1.
Devido à grande importância da atividade de extração do quartzito na região de Ouro Preto, esse trabalho teve, por objetivo, caracterizar as areias provenientes das frentes de lavra, que, normalmente, são jogadas nas encostas, visando a determinar possíveis aplicações para as mesmas e, dessa forma, garantir a reabilitação da área degradada, bem como garantir a continuidade da lavra pelos pequenos mineradores que atuam na região.

\section{Materiais e métodos}

As análises granulométricas das amostras coletadas foram efetuadas por peneiramento a seco, utilizando a série Richard de peneiras de abertura de 12,7 a $0,037 \mathrm{~mm}$. O tempo de peneiramento foi fixado em 10 minutos.

Foram efetuados ensaios preliminares de purificação das areias para a remoção das impurezas, usando separador magnético de alta intensidade (corrente de 7,8 amperes) e flotação (300 g/t de óleo de soja saponificado, $40 \%$ de sólidos em peso, pH 10, tempo de condicionamento - 5 minutos e $1200 \mathrm{rpm}$ ).
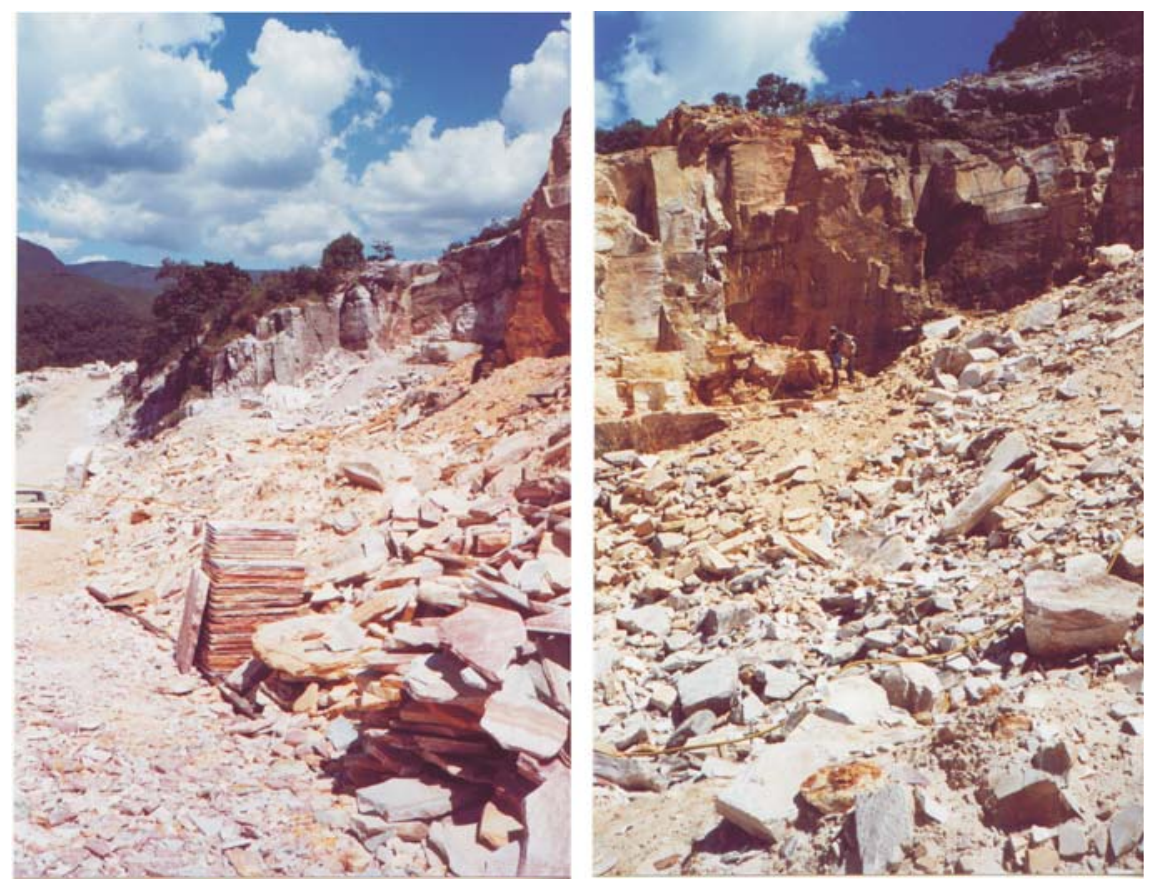

Figura 1 - Fotografias da região do morro do Taquaral de Ouro Preto/MG, mostrando a quantidade de resíduos gerados na atividade (Lima e colaboradores, 2005). 
Rosa Malena Fernandes Lima et al.

Tabela 1 - Resumo de especificações química e granulométrica de areias industriais (Barbosa \& Porphírio, 1993 e Luz \& Lins, 2005).

\begin{tabular}{|c|c|c|c|c|c|}
\hline \multirow{2}{*}{ Uso/Finalidade } & \multicolumn{4}{|c|}{ Teor (\%) } & \multirow{2}{*}{ Granulometria } \\
\hline & $\mathrm{SiO}_{2}$ & $\mathrm{Al}_{2} \mathrm{O}_{3}$ & $\mathrm{Fe}_{2} \mathrm{O}_{3}$ & Outros & \\
\hline Areia para vidro & & & & & \\
\hline Vasilhame & 92,76 & 3,779 & 0,127 & $\begin{array}{c}\mathrm{TiO}_{2}<0,034 ; \mathrm{CaO}<0,06 \\
\mathrm{MgO}<0,017 ; \quad \mathrm{K}_{2} \mathrm{O}<2,734 \\
\mathrm{Na}_{2} \mathrm{O}<0,114 ; \text { P.F. }<0,373\end{array}$ & \\
\hline Vidros Planos & $\begin{array}{c}99,5 \\
\text { (min.) }\end{array}$ & 0,30 (máx.) & 0,04 (máx.) & $\begin{array}{l}\mathrm{TiO}_{2}<0,030 ; \mathrm{Cr}_{2} \mathrm{O}_{3}<2 \mathrm{ppm} ; \\
\mathrm{MnO}_{2}<0,002 \mathrm{ppm} ; \mathrm{H}_{2} \mathrm{O}<0,05\end{array}$ & $99,5 \%>200 \#$ \\
\hline Cristal & $\begin{array}{l}98,5 \\
\text { (mín.) }\end{array}$ & 0,5 (máx.) & $\begin{array}{l}0,035 \\
\text { (máx.) }\end{array}$ & $\begin{array}{c}\mathrm{CaO}+\mathrm{MgO}<0,2 ; \mathrm{TiO}_{2}<0,03 \\
\mathrm{ZrO}_{2}<0,01 ; \mathrm{H}_{2} \mathrm{O}<0,1 \\
\mathrm{Cr}_{2} \mathrm{O}_{3}<0,001\end{array}$ & $95 \%>150 \#$ \\
\hline Fibra & $\begin{array}{l}99,0 \\
\text { (mín.) }\end{array}$ & $\begin{array}{c}0,30 \\
\text { (máx.) }\end{array}$ & 0,50 (máx.) & $\mathrm{Na}_{2} \mathrm{O}<0,1 ; \mathrm{K}_{2} \mathrm{O}<0,1, \mathrm{H}_{2} \mathrm{O}<0,5$ & $95 \%<325 \#$ \\
\hline Fundição & $88-99$ & - & - & Teor de umidade e argila total $<0,1 \%$ & 20 a $200 \#$ \\
\hline Cerâmica & 99,5 & 0,2 & 0,03 & $\begin{array}{c}\mathrm{TiO}_{2}<0,025 ; \mathrm{K}_{2} \mathrm{O}+\mathrm{Na}_{2} \mathrm{O}<0,15 ; \mathrm{CaO} \\
+\mathrm{MgO}<0,03 ; \mathrm{Cr}_{2} \mathrm{O}_{3}<3 \text { ppm; P.F. }< \\
0,1\end{array}$ & \\
\hline Esmalte & 99,5 & 0,2 & 0,03 & $\begin{array}{c}\mathrm{TiO}_{2}<0,025 ; \mathrm{K}_{2} \mathrm{O}+\mathrm{Na}_{2} \mathrm{O}<0,15 ; \mathrm{CaO} \\
+\mathrm{MgO}<0,03 ; \mathrm{Cr}_{2} \mathrm{O}_{3}<3 \text { ppm; P.F. }< \\
0,1\end{array}$ & $>40 \mu \mathrm{m}$ \\
\hline $\begin{array}{l}\text { Tinta, plástico e } \\
\text { borracha }\end{array}$ & 99,5 & 0,009 & 0,025 & $\mathrm{TiO}_{2}<0,005 ; \mathrm{CaO}<0,15$ & \\
\hline Construção civil & & & & & \\
\hline Grossa & 80 & - & - & - & 2,4 a $4,8 \mathrm{~mm}$ \\
\hline Média & & & & & 0,6 a $2,4 \mathrm{~mm}$ \\
\hline Fina & & & & & 0,15 a $0,6 \mathrm{~mm}$ \\
\hline
\end{tabular}

$11,21,5$ e $18 \%$, respectivamente, o que é extremamente interessante do ponto de vista ambiental.

No caso de aplicação para construção civil, com um corte granulométrico usando peneiras de 0,589 e 0,149 mm (areia fina), o descarte de resíduos, que são depositados nas encostas dos morros, seria reduzido de 75,4; 67 e 66,3 \% para as amostras A, B e C, respectivamente. Como areia média ( 0,80 a 2,4 mm), seria recuperado mais 3,14; 5,71 e 4,7 \%, respectivamente, para as mesmas amostras, pois, nessas granulometrias, o teor de $\mathrm{SiO}_{2}$ está acima do teor mínimo (80\%) para essa aplicação; vejam-se Tabelas 2, 3 e 4 .

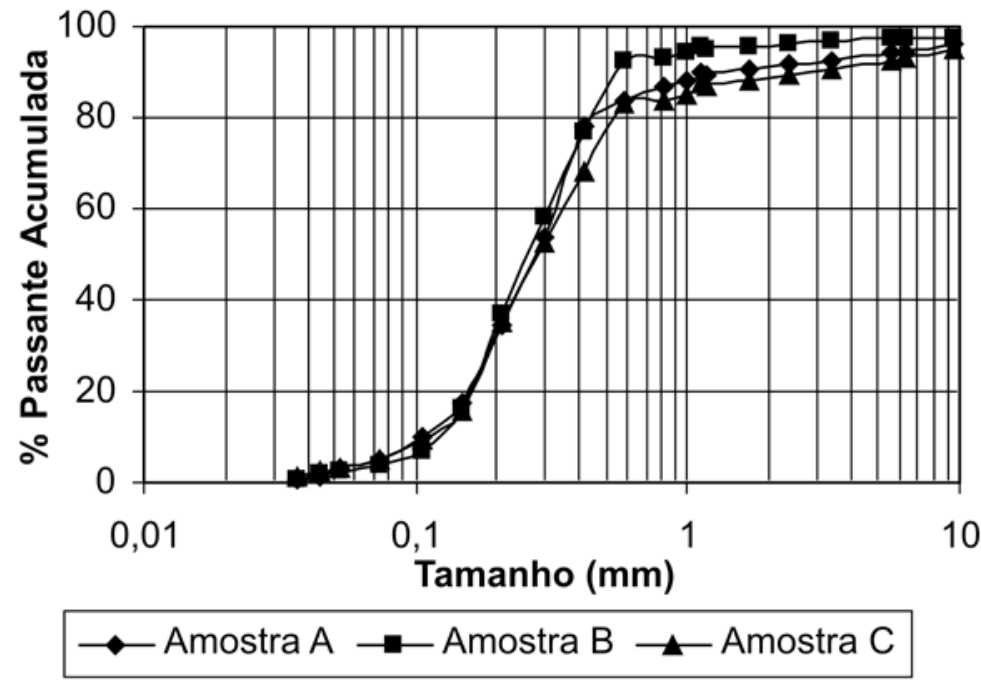

Figura 2 - Distribuições granulométricas das amostras A, B e C. 
Caracterização tecnológica de resíduos de pedreiras de quartzito da região de Ouro Preto/MG

Tabela 2 - Análise granuloquímica da amostra A.

\begin{tabular}{|c|c|c|c|c|c|c|c|c|c|c|c|c|c|c|}
\hline \multirow{2}{*}{$\begin{array}{c}\text { Tamanho } \\
\mathrm{mm}\end{array}$} & \multicolumn{10}{|c|}{ Elementos Maiores (\%) } & \multicolumn{4}{|c|}{ Elementos Menores (ppm) } \\
\hline & $\mathrm{Al}_{2} \mathrm{O}_{3}$ & $\mathrm{CaO}$ & $\mathrm{Fe}_{2} \mathrm{O}_{3}$ & $\mathrm{~K}_{2} \mathrm{O}$ & $\mathrm{Li}_{2} \mathrm{O}$ & $\mathrm{MgO}$ & $\mathrm{MnO}$ & $\mathrm{Na}_{2} \mathrm{O}$ & $\mathrm{SiO}_{2}$ & $\mathrm{Ti}_{2} \mathrm{O}$ & Co & $\mathrm{Cr}$ & $\mathrm{Pb}$ & $\mathrm{Zr}$ \\
\hline 3,36 & 3,7 & $<0,01$ & 0,35 & 0,22 & $<0,01$ & 0,01 & $<0,01$ & 0,04 & 91,1 & 0,22 & 222,5 & 38,8 & $<5,0$ & 75,2 \\
\hline 1,41 & 6,3 & 0,01 & 1,70 & 0,36 & $<0,01$ & 0,02 & $<0,01$ & 0,09 & 86,3 & 0,31 & 313,8 & 51,7 & 32,7 & 122,4 \\
\hline 1,68 & 6,1 & 0,03 & 1,38 & 0,29 & $<0,01$ & 0,02 & $<0,01$ & 0,08 & 85,9 & 0,28 & 246,4 & 39,9 & $<5,0$ & 111,5 \\
\hline 0,589 & 5,6 & 0,05 & 0,74 & 0,22 & $<0,01$ & 0,02 & $<0,01$ & 0,08 & 86,3 & 0,24 & 223,9 & 32,8 & 9,3 & 92,6 \\
\hline 0,417 & 6,6 & 0,04 & 0,39 & 0,18 & $<0,01$ & 0,01 & $<0,01$ & 0,07 & 86,2 & 0,20 & 286,2 & 31,9 & 26,5 & 79,8 \\
\hline 0,295 & 4,5 & 0,03 & 0,27 & 0,14 & $<0,01$ & 0,01 & $<0,01$ & 0,05 & 87,5 & 0,15 & 211,0 & 21,7 & $<5,0$ & 63,3 \\
\hline 0,208 & 4,1 & 0,01 & 0,11 & 0,08 & $<0,01$ & 0,01 & $<0,01$ & 0,03 & 93,6 & 0,10 & 378,7 & 18,0 & 13,9 & 48,8 \\
\hline 0,147 & 2,4 & 0,01 & 0,08 & 0,05 & $<0,01$ & $<0,01$ & $<0,01$ & 0,02 & 91,2 & 0,08 & 347,9 & 11,1 & $<5,0$ & 36,6 \\
\hline 0,104 & 1,9 & 0,01 & 0,09 & 0,04 & $<0,01$ & $<0,01$ & $<0,01$ & 0,02 & 94,3 & 0,08 & 303,2 & 9,2 & $<5,0$ & 33,2 \\
\hline 0,074 & 3,0 & 0,01 & 0,14 & 0,07 & $<0,01$ & 0,01 & $<0,01$ & 0,02 & 91,1 & 0,15 & 311,7 & 15,8 & $<5,0$ & 43,7 \\
\hline 0,038 & 5,7 & 0,01 & 0,25 & 0,21 & $<0,01$ & 0,01 & $<0,01$ & 0,05 & 85,4 & 0,34 & 289,9 & 33,3 & $<5,0$ & 88,0 \\
\hline$-0,038$ & 17,5 & 0,05 & 1,24 & 1,41 & $<0,01$ & 0,06 & $<0,01$ & 0,30 & 63,6 & 1,11 & 86,0 & 116,9 & 55,9 & 406,1 \\
\hline
\end{tabular}

Tabela 3 - Análise granuloquímica da amostra B.

\begin{tabular}{c|c|c|c|c|c|c|c|c|c|c|c|c|c|c}
\hline $\begin{array}{c}\text { Tamanho } \\
\mathbf{m m}\end{array}$ & \multicolumn{10}{|c|}{ Elementos Maiores (\% peso) } & \multicolumn{3}{c}{ Elementos Menores (ppm) } \\
\cline { 2 - 16 } & $\mathrm{Al}_{2} \mathrm{O}_{3}$ & $\mathrm{CaO}$ & $\mathrm{Fe}_{2} \mathrm{O}_{3}$ & $\mathrm{~K}_{2} \mathrm{O}$ & $\mathrm{Li}_{2} \mathrm{O}$ & $\mathrm{MgO}$ & $\mathrm{MnO}$ & $\mathrm{Na}_{2} \mathrm{O}$ & $\mathrm{SiO}_{2}$ & $\mathrm{Ti}_{2} \mathrm{O}$ & $\mathrm{Co}$ & $\mathrm{Cr}$ & $\mathrm{Pb}$ & $\mathrm{Zr}$ \\
\hline 3,36 & 4,3 & $<0,01$ & 3,7 & 0,16 & $<0,01$ & 0,01 & $<0,01$ & 0,03 & 87,4 & 0,21 & 2,283 & 0,007 & 2,586 & 0,13 \\
\hline 1,41 & 7,2 & 0,03 & 0,46 & 0,35 & $<0,01$ & 0,02 & $<0,01$ & 0,09 & 86,7 & 0,29 & 3,836 & 0,023 & 0,32 & 0,291 \\
\hline 1,68 & 7,3 & 0,01 & 1,14 & 0,29 & $<0,01$ & 0,01 & 0,01 & 0,1 & 86,9 & 0,28 & 3,874 & 0,007 & 0,794 & 0,244 \\
\hline 0,589 & 6,7 & 0,01 & 0,9 & 0,26 & $<0,01$ & 0,01 & $<0,01$ & 0,09 & 88,4 & 0,28 & 3,527 & 0,007 & 0,629 & 0,217 \\
\hline 0,417 & 7,5 & 0,02 & 0,49 & 0,22 & $<0,01$ & 0,01 & $<0,01$ & 0,08 & 86 & 0,23 & 3,961 & 0,012 & 0,341 & 0,179 \\
\hline 0,295 & 8,2 & 0,01 & 0,41 & 0,19 & $<0,01$ & 0,01 & $<0,01$ & 0,08 & 85,5 & 0,2 & 4,36 & 0,009 & 0,287 & 0,158 \\
\hline 0,208 & 5,8 & 0,01 & 0,29 & 0,15 & $<0,01$ & 0,01 & $<0,01$ & 0,06 & 87,9 & 0,16 & 3,087 & 0,006 & 0,203 & 0,121 \\
\hline 0,147 & 4,2 & 0,01 & 0,12 & 0,07 & $<0,01$ & $<0,01$ & $<0,01$ & 0,03 & 93,4 & 0,09 & 2,219 & 0,005 & 0,086 & 0,06 \\
\hline 0,104 & 3 & 0,01 & 0,14 & 0,06 & $<0,01$ & $<0,01$ & $<0,01$ & 0,02 & 95,4 & 0,08 & 1,57 & 0,004 & 0,1 & 0,049 \\
\hline 0,074 & 2,8 & 0,01 & 0,23 & 0,06 & $<0,01$ & 0,02 & $<0,01$ & 0,02 & 94,4 & 0,15 & 1,505 & 0,011 & 0,159 & 0,054 \\
\hline 0,038 & 3,7 & 0,01 & 0,22 & 0,11 & $<0,01$ & 0,01 & $<0,01$ & 0,03 & 93 & 0,17 & 1,933 & 0,006 & 0,156 & 0,092 \\
\hline$-0,038$ & 6,1 & 0,01 & 0,26 & 0,29 & $<0,01$ & 0,01 & $<0,01$ & 0,07 & 90,3 & 0,33 & 3,212 & 0,006 & 0,179 & 0,243 \\
\hline
\end{tabular}

Tabela 4 - Análise granuloquímica da amostra C.

\begin{tabular}{|c|c|c|c|c|c|c|c|c|c|c|c|c|c|c|}
\hline \multirow{2}{*}{$\begin{array}{c}\text { Tamanho } \\
\mathrm{mm}\end{array}$} & \multicolumn{10}{|c|}{ Elementos Maiores (\%) } & \multicolumn{4}{|c|}{ Elementos Menores (ppm) } \\
\hline & $\mathrm{Al}_{2} \mathrm{O}_{3}$ & $\mathrm{CaO}$ & $\mathrm{Fe}_{2} \mathrm{O}_{3}$ & $\mathrm{~K}_{2} \mathrm{O}$ & $\mathrm{Li}_{2} \mathrm{O}$ & $\mathrm{MgO}$ & $\mathrm{MnO}$ & $\mathrm{Na}_{2} \mathrm{O}$ & $\mathrm{SiO}_{2}$ & $\mathrm{Ti}_{2} \mathrm{O}$ & Co & $\mathrm{Cr}$ & $\mathrm{Pb}$ & $\overline{\mathrm{Zr}}$ \\
\hline 3,36 & 4,7 & 0,04 & 0,47 & 0,22 & $<0,01$ & 0,01 & $<0,01$ & 0,07 & 91,4 & 0,19 & 540 & 27,7 & 17 & 78,5 \\
\hline 1,41 & 6 & 0,06 & 0,75 & 0,28 & $<0,01$ & 0,01 & $<0,01$ & 0,13 & 86,1 & 0,2 & 340,1 & 29,5 & 20,8 & 80 \\
\hline 1,68 & 5,7 & 0,05 & 0,29 & 0,17 & $<0,01$ & 0,01 & $<0,01$ & 0,08 & 88 & 0,15 & 289,5 & 25,7 & $<5,0$ & 66 \\
\hline 0,589 & 4 & 0,03 & 0,11 & 0,09 & $<0,01$ & 0,01 & $<0,01$ & 0,04 & 91,4 & 0,09 & 352,7 & 15,5 & 19,7 & 47 \\
\hline 0,417 & 1,7 & $<0,01$ & 0,02 & 0,02 & $<0,01$ & $<0,01$ & $<0,01$ & 0,01 & 92,2 & 0,02 & 91,8 & 2,7 & $<5,0$ & 12 \\
\hline 0,295 & 2,8 & 0,01 & 0,05 & 0,04 & $<0,01$ & $<0,01$ & $<0,01$ & 0,02 & 96,6 & 0,08 & 331 & 11,6 & $<5,0$ & 34,9 \\
\hline 0,208 & 2,3 & 0,01 & 0,06 & 0,04 & $<0,01$ & $<0,01$ & $<0,01$ & 0,02 & 94 & 0,08 & 310,7 & 10,2 & 21,7 & 35,1 \\
\hline 0,147 & 2,9 & 0,01 & 0,09 & 0,05 & $<0,01$ & $<0,01$ & $<0,01$ & 0,02 & 92,7 & 0,16 & 317,1 & 14,6 & 19,3 & 38,4 \\
\hline 0,104 & 5,7 & 0,01 & 0,16 & 0,14 & $<0,01$ & 0,01 & $<0,01$ & 0,04 & 87,6 & 0,41 & 250,4 & 30,5 & $<5,0$ & 92,8 \\
\hline 0,074 & 9,3 & 0,02 & 0,41 & 0,46 & $<0,01$ & 0,02 & 0,02 & 0,1 & 81,2 & 0,74 & 125,7 & 54,5 & 34,3 & 267,2 \\
\hline 0,038 & 17,7 & 0,05 & 1,21 & 0,94 & $<0,01$ & 0,05 & 0,09 & 0,22 & 72,8 & 1,91 & 71,7 & 109,3 & 50,2 & 735,3 \\
\hline$-0,038$ & 31,1 & 0,09 & 2,32 & 1,09 & $<0,01$ & 0,08 & 0,17 & 0,28 & 51 & 3,28 & 99,3 & 188,2 & 129,7 & 1165,8 \\
\hline
\end{tabular}

666 REM: R. Esc. Minas, Ouro Preto, 60(4): 663-668, out. dez. 2007 
Rosa Malena Fernandes Lima et al.

A recuperação em massa obtida na purificação da amostra A, usando separação magnética de alta intensidade, foi de 89\%. Nas Tabelas 5 e 6 estão apresentadas as análises granuloquímicas das areias purificadas (amostra A e B), utilizando separação magnética de alta intensidade (corrente de 7,8 A). Observa-se, pela Tabela 5, que os teores de impurezas dos concentrados são maiores nas faixas granulométricas acima de $589 \mu \mathrm{m}$ (28 \#) e abaixo de $147 \mu \mathrm{m}$ (100 \#). Esse mesmo comportamento já era observado na amostra de cabeça (Tabela 2). Através desse ensaio, observa-se, também, que o teor de $\mathrm{TiO}_{2}$ está acima do limite permitido para a indústria de vidro para todas as faixas granulométricas analisadas. No caso da amostra B
(Tabela 6), os teores de $\mathrm{TiO}_{2}$, para a faixa granulométrica entre $417 \mu \mathrm{m}$ (35 \#) e $208 \mu \mathrm{m}$ (65 \#), encontram-se dentro das especificações para vidro (teor máximo permitido de 0,03\%), o que é bastante significativo em termos de massa recuperada.

Na Tabela 7, estão apresentados os balanços de massa dos ensaios de purificação das três amostras, bem como os teores de sílica e de impurezas nos concentrados. A recuperação em massa (produto afundado), para todas as três amostras testadas, estava acima de 93\% com teores de sílica acima de 90\%. Quanto aos teores de impurezas, observa-se que foi obtido um concentrado (produto afundado) dentro das especificações para vidro (vasilhame) para a amostra A.
No caso da amostra $\mathrm{C}$, o teor de $\mathrm{TiO}_{2}$ no concentrado foi de 0,05 \%, que está ligeiramente fora das especificações para vidro.

Através desses ensaios preliminares de purificação das areias estudadas por separação magnética de alta intensidade e por flotação, verifica-se a possibilidade de obtenção de concentrados dentro das especificações para vidro, especialmente para a purificação por flotação, pois há de se salientar que os resultados dos teores de sílica e de impurezas foram obtidos para amostras globais dos concentrados, o que não foi obtido para o concentrado da separação magnética de alta intensidade, exceto para a amostra B para a faixa granulométrica de 208 a $417 \mu \mathrm{m}$.

Tabela 5 - Análise granuloquímica do produto não magnético da amostra A (corrente de 7,8 A).

\begin{tabular}{|c|c|c|c|c|c|c|c|c|c|c|c|}
\hline \multicolumn{2}{|c|}{ Tamanho } & \multirow{2}{*}{$\begin{array}{c}\begin{array}{c}\text { Peso } \\
\text { (\%) }\end{array} \\
\end{array}$} & \multicolumn{9}{|c|}{ Teores (\%) } \\
\hline$(\#)$ & $\mu \mathrm{m}$ & & $\mathrm{SiO}_{2}$ & $\mathrm{Al}_{2} \mathrm{O}_{3}$ & $\mathrm{Fe}_{2} \mathrm{O}_{3}$ & $\mathrm{TiO}_{2}$ & $\mathrm{CaO}$ & $\mathrm{MgO}$ & $\mathrm{K}_{2} \mathrm{O}$ & $\mathrm{Na}_{2} \mathrm{O}$ & P.F. \\
\hline 28 & 589 & 6,4 & 93,8 & 5,45 & 0,1 & 0,16 & 0,07 & 0,012 & 0,14 & 0,076 & 0,22 \\
\hline 35 & 417 & 11,8 & 97,2 & 2,45 & 0,05 & 0,07 & 0,05 & 0,007 & 0,07 & 0,039 & 0,08 \\
\hline 48 & 295 & 21 & 98,4 & 1,36 & 0,04 & 0,06 & 0,04 & 0,006 & 0,05 & 0,031 & 0,07 \\
\hline 65 & 208 & 21,4 & 98,3 & 1,41 & 0,06 & 0,07 & 0,04 & 0,006 & 0,04 & 0,03 & 0,08 \\
\hline 100 & 147 & 22,8 & 97,8 & 1,75 & 0,07 & 0,11 & 0,07 & 0,015 & 0,05 & 0,033 & 0,11 \\
\hline 200 & 74 & 13,2 & 94,7 & 4,23 & 0,12 & 0,31 & 0,12 & 0,012 & 0,14 & 0,052 & 0,28 \\
\hline-200 & - & 3,4 & 82,6 & 15,56 & 0,34 & 1,05 & 0,29 & 0,03 & 0,19 & 0,067 & 0,86 \\
\hline \multicolumn{2}{|c|}{ Total Recalculado } & 100 & 96,8 & 2,71 & 0,08 & 0,15 & 0,07 & 0,01 & 0,07 & 0,039 & 0,15 \\
\hline
\end{tabular}

Tabela 6 - Análise granuloquímica do produto não magnético da amostra B (corrente de 7,8 A).

\begin{tabular}{|c|c|c|c|c|c|c|c|c|c|c|c|}
\hline \multicolumn{2}{|c|}{ Tamanho } & \multirow{2}{*}{$\begin{array}{c}\text { Peso } \\
\text { (\%) }\end{array}$} & \multicolumn{9}{|c|}{ Teores (\%) } \\
\hline (\#) & $\mu \mathrm{m}$ & & $\mathrm{SiO}_{2}$ & $\mathrm{Al}_{2} \mathrm{O}_{3}$ & $\mathrm{Fe}_{2} \mathrm{O}_{3}$ & $\mathrm{TiO}_{2}$ & $\mathrm{CaO}$ & $\mathrm{MgO}$ & $\mathrm{K}_{2} \mathrm{O}$ & $\mathrm{Na}_{2} \mathrm{O}$ & P.F. \\
\hline 28 & 589 & 9,1 & 95,3 & 3,87 & 0,16 & 0,07 & 0,05 & 0,008 & 0,15 & 0,05 & 0,3 \\
\hline 35 & 417 & 10,9 & 97,3 & 2,32 & 0,1 & 0,02 & 0,03 & 0,002 & 0,06 & 0,02 & 0,1 \\
\hline 48 & 295 & 18,7 & 98,4 & 1,35 & 0,08 & 0,02 & 0,01 & 0,002 & 0,04 & 0,01 & 0,05 \\
\hline 65 & 208 & 19,3 & 98,5 & 1,25 & 0,1 & 0,03 & 0,01 & 0,002 & 0,04 & 0,01 & 0,09 \\
\hline 100 & 147 & 23,6 & 98,2 & 1,48 & 0,1 & 0,06 & 0,02 & 0,003 & 0,04 & 0,01 & 0,08 \\
\hline 200 & 104 & 15,1 & 95,7 & 3,29 & 0,14 & 0,21 & 0,17 & 0,008 & 0,1 & 0,03 & 0,35 \\
\hline-200 & - & 3,3 & 81,8 & 14,4 & 0,57 & 0,86 & 0,71 & 0,004 & 0,3 & 0,09 & 1,28 \\
\hline \multicolumn{2}{|c|}{ Total Recalculado } & 100 & 97 & 2,42 & 0,12 & 0,09 & 0,07 & 0,003 & 0,07 & 0,02 & 0,18 \\
\hline
\end{tabular}


Caracterização tecnológica de resíduos de pedreiras de quartzito da região de Ouro Preto/MG

Tabela 7 - Balanço de massa dos ensaios de flotação das amostras A, B e C.

\begin{tabular}{c|c|c|c|c|c|c|c|c|c}
\hline \multirow{2}{*}{ Amostra } & \multirow{2}{*}{ Produto } & \multicolumn{2}{|c|}{ Massa } & \multicolumn{7}{c}{ Teores (\%) } \\
\cline { 3 - 10 } & & $\mathbf{( g )}$ & $\mathbf{( \% )}$ & $\mathbf{S i O}_{2}$ & $\mathbf{A l}_{2} \mathbf{O}_{3}$ & $\mathbf{F}_{2} \mathbf{O}_{3}$ & $\mathbf{C r}^{*}$ & $\mathbf{C o}^{*}$ & $\mathbf{T i O}_{2}$ \\
\hline \multirow{3}{*}{$\mathbf{A}$} & Flotado & 50,7 & 6,5 & 79,7 & 4,7 & 10,7 & 61,5 & 995 & 0,33 \\
& Afundado & 730,0 & 93,5 & 98,8 & 1,0 & 0,112 & 5,46 & 205 & 0,01 \\
& Total & 780,7 & 100,0 & 97,7 & 1,9 & 4,1 & 9,1 & 256,4 & 0,031 \\
\hline \multirow{3}{*}{$\mathbf{B}$} & Flotado & 43,9 & 5,6 & 78,9 & 9,9 & 10,6 & 131 & 107 & - \\
& Afundado & 745,0 & 94,4 & 90,3 & 5,7 & 3,56 & 49,4 & 9 & 0,25 \\
& Total & 788,9 & 100,0 & 89,7 & 6,0 & 3,95 & 53,97 & 14,49 & 0,236 \\
\hline \multirow{3}{*}{$\mathbf{C}$} & Flotado & 29,8 & 3,8 & 85,1 & 7,2 & 4,07 & 113 & 107 & 0,24 \\
& Afundado & 755,0 & 96,2 & 95,5 & 3,3 & 0,22 & 16 & 2 & 0,05 \\
& Total & 784,8 & 100,0 & 95,1 & 3,5 & 0,37 & 19,69 & 5,99 & 0,06 \\
\hline
\end{tabular}

${ }^{*} \mathrm{ppm}$

\section{Conclusões}

- As curvas de distribuição granulométrica das três amostras analisadas mostram-se bastante similares com $\mathrm{d}_{80}$ da amostra A e B igual a 0,35 mm e da amostra C igual a 0,42 mm.

- Observou-se que há a possibilidade de redução de 82, 78 e 89 \%, respectivamente, dos resíduos descartados nas encostas dos morros (amostras A, B e C), fazendo somente um corte granulométrico entre 0,589 e 0,074mm, pois os teores de $\mathrm{SiO}_{2}$ das amostras A, B e C foram de 89,94; 92,74 e $92,77 \%$, respectivamente. Esses valores estão dentro das especificações de areia para aplicação em fundição.

- Uma outra possibilidade de aplicação dos resíduos de quartzito é como areia fina (0,589 e 0,149 mm) e média (0,8 a 2,4 mm), o que acarretaria uma redução de resíduos depositados nas encostas dos morros em torno de 74,5; 73 e $71 \%$, respectivamente, das amostras A, B e C.
- Verificou-se a possibilidade de obtenção de areia para aplicação em vidro de vasilhame após a purificação das mesmas.

- A purificação das areias, usando flotação das impurezas, mostrou-se mais eficaz do que a separação magnética de alta intensidade, pois, nesse caso, embora tenha ocorrido a redução dos teores de $\mathrm{Fe}_{2} \mathrm{O}_{3}$ e $\mathrm{Al}_{2} \mathrm{O}_{3}$, para níveis aceitáveis para vidro, a obtenção de teor de $\mathrm{TiO}_{2}$, dentro dos limites estabelecidos, para essa finalidade mostrou-se bastante complicada.

\section{Agradecimentos}

Os autores agradecem à Fapemig pelo financiamento do projeto.

\section{Referências bibliográficas}

BARBOSA, M. I. M., PHORPHÍRIO, N. H. Projeto caracterização tecnológica de lascas de quartzo. Centro de Tecnologia Mineral - CETEM/CNPq. Relatório Técnico. 1993. $106 \mathrm{p}$.

LIMA, R. M. F., SILVA, A. F. S., LUZ, J. A. M., OLIVEIRA, M. L. M. Estudo para o aproveitamento econômico dos resíduos de pedreiras de quartzito. Relatório Técnico. 2005. 126 p.

LIMA, R. M. F., SILVA, F. A. S., JORDT-EVANGELISTA, H. Uso de resíduos de pedreira de quartzito como areia para construção civil e fundição. In: ENCONTRO NACIONAL DE TRATAMENTO DE MINÉRIOS E METALURGIA EXTRATIVA, 21. Anais... Natal-RN, 20 a 24 de novembro de 2005. p.351-357.

LUZ, A. B. LINS, F.A. F. Areia Industrial. In: Rochas e Minerais Industriais: usos e especificações. LUZ, A. B, LINS, F. A. (Ed.). Rio de Janeiro: CETEM/MCT, 2005. p.107 a 126. 2005.

Artigo recebido em 08/11/2006 e aprovado em 26/06/2007.

\section{REM - Revista Escola de Minas www.rem.com.br}

